\title{
Hypotonic versus isotonic intravenous maintenance fluids in critically ill pediatric patients: a randomized clinical trial
}

\author{
Facundo A. Jorro Barón, M.D., ${ }^{a}$ Claudia N. Meregalli, M.D., ${ }^{a}$ \\ Valeria A. Rombolá, M.D., ${ }^{a}$ Cecilia Bolasell, M.D., ${ }^{a}$ Vilma E. Pigliapoco, Pharmacist ${ }^{b}$ \\ Silvia E. Bartoletti, Pharmacist, ${ }^{b}$ and Gustavo E. Debaisi, M.D. ${ }^{a}$
}

\begin{abstract}
Introduction. Hypotonic fluids have been associated with the development of iatrogenic hyponatremia.

Objectives. To assess variations in serum sodium (sNa) following the intravenous administration of isotonic maintenance fluids $(0.9 \% \mathrm{NaCl} / 5 \%$ dextrose) compared to hypotonic maintenance fluids $(0.45 \% \mathrm{NaCl} / 5 \%$ dextrose)

Material and Methods. Randomized, controlled, double-blind clinical trial. Pediatric patients with an expected length of stay in the intensive care unit of more than 24 hours were enrolled, with normal serum $\mathrm{Na}$, and IV fluids $>80 \%$ of total maintenance fluids. Serum Na level was measured before administering maintenance fluids and when reducing the administration to $<80 \%$ of total fluids.

Results. The study included 63 patients who were randomly assigned to receive hypotonic $(n=32)$ or isotonic $(n=31)$ maintenance fluids. Baseline characteristics were similar in both groups. There were no differences in terms of volume of fluid administered (hypotonic group: $865 \pm 853 \mathrm{~mL}$; isotonic group: $778 \pm 649 \mathrm{~mL}$; $\mathrm{p}=0.654$ ) or infusion duration (hypotonic group: $24 \pm 10.8$ hours; isotonic group: $27.6 \pm 12.8$ hours; $\mathrm{p}=0.231$ ). A difference was found in the serum Na following the administration of maintenance fluids (hypotonic group: $137.8 \pm 4.3 \mathrm{mmol} / \mathrm{L}$; isotonic group: $140.0 \pm 4.1 \mathrm{mmol} / \mathrm{L}, \mathrm{p}=0.04$ ). None of these two maintenance fluids increased the risk of hyponatremia $(\mathrm{Na}<135 \mathrm{mmol} / \mathrm{L})$ or hypernatremia ( $\mathrm{Na}>145 \mathrm{mmol} / \mathrm{L})$.
\end{abstract}

a. Pediatric Intensive, Care Unit.

b. Pharmacy Unit. Hospital de Niños "Pedro de Elizalde" Buenos Aires City

E-mail address: Facundo Jorro Barón, M.D.: jorrobox@yahoo.com.ar

Registered in

ClinicalTrials.gov (NCT01301274),

February 18, 2011.

Conflict of interest:

None.

Received: 12-7-2012 Accepted: 3-22-2013 maintenance fluids could be similar to those of breast milk ( $\mathrm{NaCl} 10-$ $30 \mathrm{mmol} / \mathrm{L}$ ). Hypotonic maintenance fluids in healthy children should not be associated with any risk, but they could have harmful consequences in ill children with impaired free water excretion.

Since the 1980s, numerous publications have described the development of severe neurological morbidity and death secondary to iatrogenic hyponatremia. ${ }^{2-4}$ In spite of the quality and volume of infusions administered, serum sodium concentration is usually within normal limits (135-145 $\mathrm{mmol} / \mathrm{L}$ ).

Water and sodium homeostasis is one of the major functions of the kidneys through free water excretion. Several stimuli have been described for antidiuretic hormone secretion; many of them are common in critically ill patients, such as respiratory and digestive tract infections, pain, nausea, opioid use, and the post-surgical period. ${ }^{5-7}$ Therefore, maintenance fluids containing large quantities of free water could induce iatrogenic hyponatremia in these patients. Due to an increased number of reports of iatrogenic hyponatremia cases related to permanent brain injury and brain death, several authors currently recommend the use of isotonic fluids as maintenance therapy for pediatric patients. ${ }^{8-11}$ In spite of the growing evidence that indicates that hypotonic fluids increase the risk of hyponatremia, there are few prospective clinical trials confirming such risk in pediatric patients. ${ }^{8}$ In Argentina, some facilities still use maintenance fluids containing 0.18 - 
$0.3 \% \mathrm{NaCl}(30-45 \mathrm{mmol} / \mathrm{L})$ in $5 \%$ dextrose with no control of serum $\mathrm{Na}$ at the beginning of or during the infusion. ${ }^{12}$

The main objective of this clinical trial was to assess the difference in serum $\mathrm{Na}$ using two maintenance fluids of different tonicity, isotonic maintenance fluid (IMF) $(0.9 \% \mathrm{NaCl} / 5 \%$ dextrose) and hypotonic maintenance fluid (HMF) $(0.45 \% \mathrm{NaCl} / 5 \%$ dextrose) for less than 48 hours following admission to a Pediatric Intensive Care Unit (PICU).

\section{MATERIAL AND METHODS Protocol}

A prospective, controlled, double-blind, randomized clinical trial was conducted at the PICU of the Hospital General de Niños "Pedro de Elizalde" (HGNPE) between February 1 and October 31, 2011. Eligibility criteria included children from 1 month to 18 years old with an expected length of stay at the PICU of more than 24 hours, with normal serum Na (135$145 \mathrm{mmol} / \mathrm{L}$ ) measured at the time of admission to the PICU or after IV fluid resuscitation, and requiring $>80 \%$ of IV fluids out of the total maintenance fluid administration. Patients were excluded if they had previous kidney failure (serum creatinine $>1 \mathrm{mg} / \mathrm{dL}$ in children $\leq 3$ years old, and serum creatinine $>1.5 \mathrm{mg} / \mathrm{dL}$ in children $>3$ years old), liver failure with ascites and portal hypertension, adrenal insufficiency, nephrotic or nephritic syndrome, Kawasaki disease, sickle cell disease, diabetes insipidus, congenital metabolic disease, plasma glucose $>200 \mathrm{mg} / \mathrm{dL}$, required total parenteral nutrition or hyperhydration (patients receiving cancer treatment or with risk of tumor lysis syndrome), and those re-admitted to the PICU. Patients in whom a second sample of serum $\mathrm{Na}$ was not obtained were excluded from the study.

Once parents provided their informed consent, children were randomly assigned to two groups: the hypotonic group (HMF), who received maintenance fluids with a $\mathrm{Na}$ concentration of $77 \mathrm{mmol} / \mathrm{L}$, and the isotonic group (IMF), who received maintenance fluids with a $\mathrm{Na}$ concentration of $154 \mathrm{mmol} / \mathrm{L}$. The addition of up to $20 \mathrm{mmol} / \mathrm{L}$ of potassium to maintenance fluids of both groups was the treating physician's decision. Glucose concentrations were the same in both groups ( $5 \%$ dextrose). Maintenance fluids were prepared and masked by research pharmacists. Maintenance fluids were prepared under a class $2 \mathrm{X}$ biosafety laminar vertical flow and stored in tight individual bags at a temperature of $2-8^{\circ} \mathrm{C}$ until they were dispensed with the corresponding randomization code. Fluid volume was estimated using the HGNPE's standards: $1500 \mathrm{~mL} / \mathrm{m}^{2}$ if the patient's weight was $>10 \mathrm{~kg}$, and $80 \mathrm{~mL} / \mathrm{kg}$ of the patient's weight was $\leq 10 \mathrm{~kg}$, every 24 hours.

The treating physician could discontinue treatment with maintenance fluids and withdraw the patient from the protocol at any time if he/she considered that it was necessary to change the $\mathrm{Na}$ infusion concentration. Treating physicians were aware of sodium concentration results of patients enrolled in the study in order to manage adverse events related to serum $\mathrm{Na}$ alterations.

The primary outcome measure was the change in serum $\mathrm{Na}(\mathrm{mmol} / \mathrm{L})$ between the baseline and the final value produced by the maintenance fluid infusion. Serum Na was measured before randomization and when patients reached $>20 \%$ of the total oral fluid intake or after 48 hours of starting the maintenance fluid infusion, because most PICU patients usually achieve a complete enteral nutrition in the first 48 hours of hospitalization or when parenteral nutrition is prescribed. Serum $\mathrm{Na}$ and glucose levels were determined using a blood gas and electrolyte analyzer (Gem Premier 3000, Instrumentation Laboratory, Lexington, MA, USA). Values were considered as follows: normal serum $\mathrm{Na}$ level: 135-145 mmol/L, hyponatremia: $<135 \mathrm{mmol} / \mathrm{L}$, severe hyponatremia: $<130 \mathrm{mmol} / \mathrm{L}$; and hypernatremia $>145 \mathrm{mmol} / \mathrm{L}$.

Secondary outcome measures included 28 day mortality after randomization, number of days without mechanical ventilation at 28 days since the admission to the PICU, length of stay in the PICU (days), presence of hyponatremia and hypernatremia, and number of patients with severe hyponatremia.

Two external observers reviewed the results once half of the population had been recruited; no risks were found that would prevent the study completion.

This study was approved by the Institutional Review Board of the Teaching and Research Department and the Bioethics Committee (NCT01301274).

\section{Sample size calculation}

The sample size was calculated based on the primary outcome of the study. The minimum serum Na reduction was estimated at $2 \mathrm{mmol} / \mathrm{L}$ in patients on hypotonic maintenance fluids, with 
a standard deviation of $2 \mathrm{mmol} / \mathrm{L} .{ }^{12}$ Considering an alpha error of 0.05 and a $90 \%$ power to detect such difference, the sample size was estimated at 30 patients per group plus patients lost to followup.

\section{Randomization}

Patients were assigned to HMF or IMF administration according to a computergenerated randomization list (Microsoft Excel 2007) in a four block design. The randomization code was maintained by the research pharmacist and kept blinded to all staff members in charge of indicating and prescribing the maintenance fluids. These were prepared and coded at the pharmacy, and all participants, nurses, medical and research staff members, researchers (except pharmacists) and members of the safety monitoring committee were unaware of sodium concentration in administered fluids.

\section{Statistical analysis}

Continuous data were expressed as mean \pm standard deviation (SD) or as median and interquartile (IQ) range, and they were compared using the Student's t test or the Mann-Whitney $\mathrm{U}$ test (non parametric data). Categorical data were presented as rates and compared using an $\chi^{2}$ test or Fisher's exact test. The significance level during the study was established at $<0.05$. Results were analyzed using the STATA 9.0 software for Windows (Copyright 1984-2003 Stata Corporation 4905 Lakeway Drive College Station, TX, USA).

\section{RESULTS}

Between February 1 and October 31, 2011, 251 patients were admitted to the HGNPE's PICU; 94 patients were eligible for this study; and finally 66 pediatric patients were randomized. It was not possible to obtain a second measurement of serum $\mathrm{Na}$ in three patients and they were excluded from the final data analysis. A total of 63 patients were included (Figure 1). Thirty two patients were randomly assigned to hypotonic maintenance fluids, and 31 to isotonic maintenance fluids.

There were no differences between groups in terms of their baseline characteristics: age, gender, weight, Pediatric Index of Mortality 2 (PIM2),

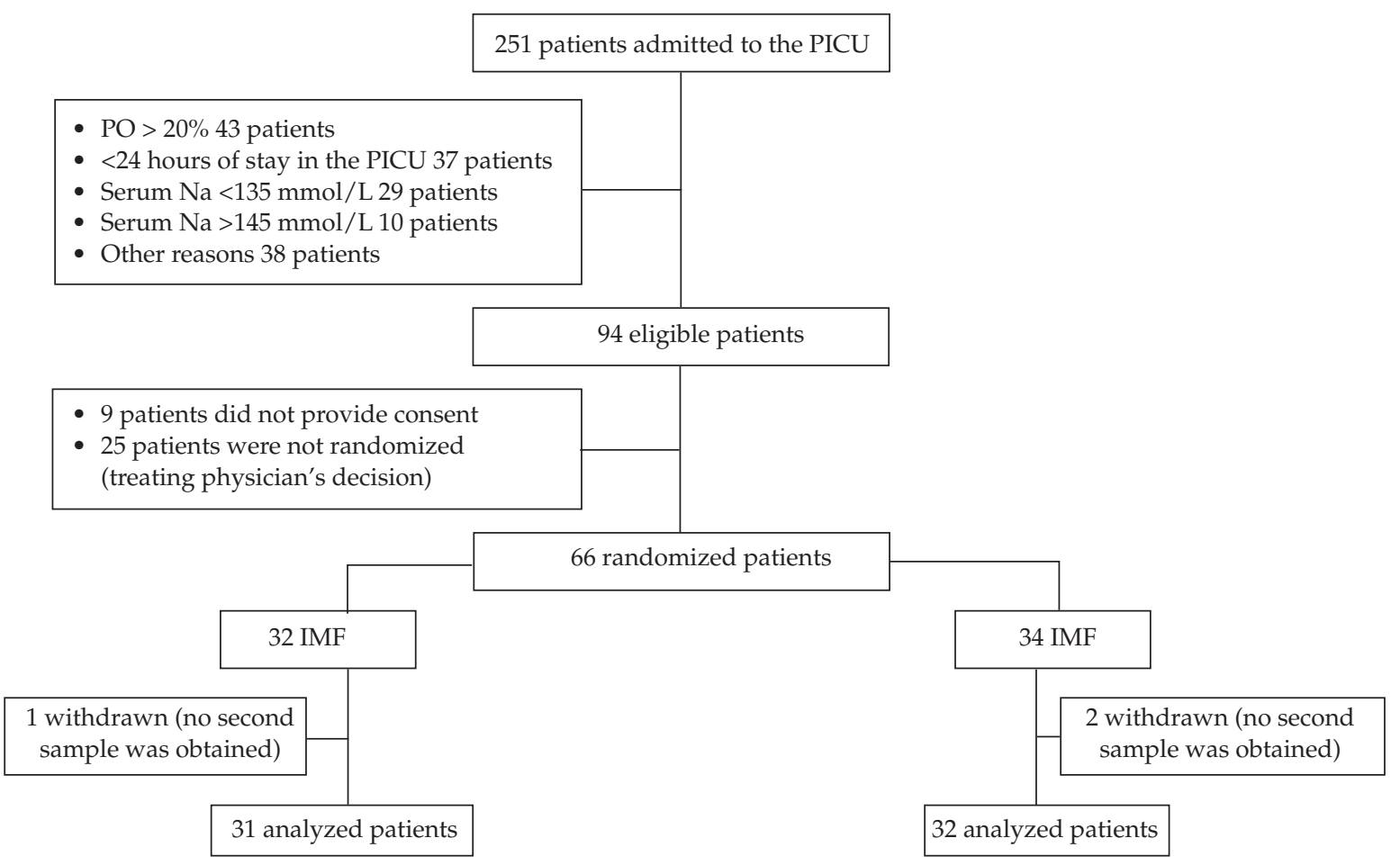

IMF: isotonic maintenance fluid; HMF: hypotonic maintenance fluid. PO: oral fluids. 
condition (clinical or surgical), and serum $\mathrm{Na}$ (Table 1).

There were also no differences between the groups in relation to the volume and duration of the maintenance fluid administration (Table 2). Patients received less maintenance fluids than the standard maintenance rate $(100 \mathrm{~mL} / \mathrm{kg} /$ day with a weight of up to $10 \mathrm{~kg}$ ): $37 \%$ less in the HMF group and $23 \%$ less in the IMF group. ${ }^{1}$

Following infusion, a significant difference in serum $\mathrm{Na}$ was observed between groups: IMF $140 \pm 4.1$ versus HMF $137.8 \pm 4.3 \mathrm{mmol} / \mathrm{L}$, $\mathrm{p}=0.04$. The absolute change in the serum sodium concentration was higher in patients receiving HMF (-1.8 $\mathrm{mmol} / \mathrm{L}$ [IQ $-0.1,-3.66])$ versus patients receiving IMF $(0.9 \mathrm{mmol} / \mathrm{L}$ [IQ -2.8 , 1.1]); however, this difference was not significant.

There were no difference between groups in the number of days without mechanical ventilation, length of stay in the PICU, and 28 day mortality. The three patients who died and received hypotonic maintenance fluids maintained a sodium concentration above 130 $\mathrm{mmol} / \mathrm{L}$ throughout the study, and the cause of death apparently was not related to the maintenance fluid infusion (Table 3).

TABLE 1. Characteristics of patients at the beginning of the study

\begin{tabular}{lcccc}
\hline & Total & HMF & IMF & $p$ \\
\hline Randomized patients (n) & 63 & 32 & 31 & $19(61.3)$ \\
Males (n) (\%) & $37(58.7)$ & $18(56.2)$ & $5(3,10)$ & 0.80 \\
Age, median (IQ), (months) & $5(3,9)$ & $4.5(3,9)$ & $6(5,10)$ & 0.54 \\
Weight, median (IQ), (kg) & $6(5,11)$ & $6.1(5,16)$ & $2.5(1.6,7)$ & 0.35 \\
PIM2, median (IQ) & $2.61(6,7)$ & $4.4(1.4,8.5)$ & & 0.73 \\
\hline Type of diagnosis & & & $30(93.5)$ & 0.71 \\
Clinical (total) (\%) & $59(89)$ & $29(85)$ & $22(68)$ & 0.41 \\
Respiratory disease & $40(60)$ & $18(53)$ & $2(6.5)$ & 1 \\
Hematooncological disease & $4(6)$ & $2(6)$ & $2(12.5)$ & 0.73 \\
Infectious disease & $9(14)$ & $4(15)$ & $2(6.5)$ & 0.42 \\
Neurological disease & $6(9)$ & $5(15)$ & $139.2 \pm 3.5$ & 0.42 \\
Surgical (total) (\%) & $7(11)$ & $139.6 \pm 3.2$ & 0.63 \\
Baseline serum Na (mean \pm SD) (mmol/L) & $139.4 \pm 3.4$ & & \\
\hline
\end{tabular}

PIM2: Pediatric Index of Mortality 2; serum Na: serum sodium concentration; HMF: hypotonic maintenance fluid; IMF: isotonic maintenance fluid; IQ: interquartile.

TABLE 2. Volume of fluid administered, changes in serum sodium concentration, and patients' characteristics at end of study

\begin{tabular}{lccc}
\hline & HMF (n= 32) & IMF (n= 31) & $p$ \\
\hline Administered fluids (mL) median (IQ) & $577(332,987)$ & $594(336,900)$ & 0.92 \\
Hours of MF administration (median \pm SD) & $24 \pm 10.8$ & $27.6 \pm 12.8$ & 0.23 \\
Rate (expressed as a \% of the traditional & & & \\
maintenance fluid administration rate) & 63 & $140 \pm 4.1$ & 0.04 \\
Final serum Na (mmol/L) (mean \pm SD) & $137.8 \pm 4.3$ & $21,5 \pm 6.6$ & 0.40 \\
Days without mechanical ventilation (mean \pm SD) & $19.9 \pm 7.9$ & $12 \pm 11.5$ & 0.13 \\
Length-of-stay in the PICU (days) (mean \pm SD) & $8.5 \pm 5.5$ & $0(0)$ & 0.08 \\
Mortality (n) (\%) & $3(9.4)$ & $1(3.3)$ & 1 \\
Patients with hypernatremia (n) (\%) & $2(6.7)$ & $4(12.9)$ & 1 \\
Patients with hyponatremia (n) (\%) & $5(15.5)$ & & \\
\hline
\end{tabular}

Serum Na: serum sodium concentration; HMF: hypotonic maintenance fluids; IMF: isotonic maintenance fluids; IQ: interquartile; PICU: Pediatric Intensive Care Unit; MF: maintenance fluids. 
None of the maintenance fluids caused an increase in the risk of hyponatremia (HMF: RR 1.21; 95\% CI 0.36-4.12; IMF: RR 0.83; 95\% CI 0.242.81) or hypernatremia (HMF: RR $1.93 ; 95 \% \mathrm{CI}$ 0.19-19.69; IMF: RR 0.52; 95\% CI 0.05-5.24).

There was only one case of severe hyponatremia (serum $\mathrm{Na}<130 \mathrm{mmol} / \mathrm{L}$ ) in a patient receiving HMF.

\section{DISCUSSION}

The results obtained in this clinical trial showed that hypotonic maintenance fluids caused a higher reduction in serum $\mathrm{Na}$, while isotonic maintenance fluids did not produce any alteration in serum $\mathrm{Na}$ of critically ill pediatric patients. These results were similar to those observed in a clinical trial conducted by Álvarez Montañana, et al. ${ }^{13}$ and confirmed by a metaanalysis ${ }^{11}$ indicating that the administration of a hypotonic maintenance fluid is a significant risk factor for iatrogenic hyponatremia in children.

A recent study demonstrated a reduction of hyponatremia in children after surgery receiving an isotonic maintenance fluid, with no development of iatrogenic hypernatremia. In this study, elevated levels of antidiuretic hormone $(\mathrm{ADH})$ were observed in the first 24 hours following non osmotic stimuli for ADH secretion. ${ }^{14}$

Reduction in serum Na could be explained by a sodium deficit or a positive electrolyte-free water (EFW) balance. High ADH levels impact EFW secretion, and several non osmotic stimuli have been shown to increase ADH secretion. ${ }^{5,8,14,15}$ Both maintenance fluids administered to the patients in our study contained enough sodium to meet daily demands, ${ }^{1}$ for this reason, reduced serum $\mathrm{Na}$ could be attributed to the excess EFW intake in hypotonic maintenance fluids. In our study, the absolute value of serum $\mathrm{Na}$ reduction was not clinically significant because sodium concentration levels were below
$130 \mathrm{mmol} / \mathrm{L}$ in just one patient, who had no clinical manifestations.

However, taking into consideration that patients in our study received a lower volume of maintenance fluids than that described by Holliday and Segar in 1959 (37\% lower for HMF and $23 \%$ lower for IMF), serum Na reduction could have been more relevant if $100 \%$ of the volume of maintenance fluids traditionally proposed had been administered. ${ }^{1}$ Álvarez Montañana, et al. suggested that the possibility of developing hyponatremia with an hypotonic maintenance fluid infusion is cumulative depending on the infusion duration. ${ }^{13}$

Kannan, et al. observed a lower incidence of hyponatremia when patients received $75 \%$ of the standard maintenance rates versus standard rates. ${ }^{16}$ Hypotonic maintenance fluids at high rates have been associated with morbidity and mortality in pediatric patients due to iatrogenic hyponatremia..$^{4,17-20}$ Some authors suggest that the use of a lower volume of maintenance fluids would be equal to or even more effective to prevent hospital-acquired hyponatremia than the administration of isotonic maintenance fluids. ${ }^{12,13,21}$ Neville, et al. found that the risk of hyponatremia was reduced with the use of isotonic maintenance fluids, but this did not occur when the volume of administered maintenance fluids was restricted. ${ }^{22}$ The present study was not intended to compare variations in serum $\mathrm{Na}$ caused by the administration of different volumes of maintenance fluids.

The principal limitation of this study was that ADH levels could not be measured. However, the increase of ADH levels in hospitalized pediatric patients has been described in different studies. ${ }^{5,8,14,15}$ In our study, few patients were withdrawn and in all a serum Na measurement was made at baseline. In many of the clinical trials cited here serum $\mathrm{Na}$ was not measured before the administration of maintenance fluids, and this could have resulted in an over-estimation

TABLE 3. Characteristics of deceased patients

\begin{tabular}{|c|c|c|c|c|c|}
\hline Age (months) & Diagnosis & Baseline serum $\mathrm{Na}$ & Final serum $\mathrm{Na}$ & Date of death & Cause of death \\
\hline Acu & e lymphoblastic leukemia & 135 & 130 & 24 & Septic shock \\
\hline 1 & Meningitis & 144 & 140 & 4 & Brain death \\
\hline \multirow[t]{2}{*}{2} & Infection caused by & & & & \\
\hline & Bordetella pertussis & 136 & 142 & 2 & Cardiogenic shock \\
\hline
\end{tabular}

All patients received hypotonic maintenance fluids.

Serum Na: serum sodium concentration. 
of hyponatremia caused by maintenance fluids at the end of the trial. In our study, no patients with serum sodium levels below $135 \mathrm{mmol} / \mathrm{L}$ were included, and this is probably the reason why we have not seen clinical manifestations of hyponatremia or more patients with hyponatremia when finishing the study.

Only three patients had a serum sodium concentration higher than $145 \mathrm{mmol} / \mathrm{L}$, which was not considered a risk $(<150 \mathrm{mmol} / \mathrm{L})$. Due to our patients' critical condition, it was difficult to estimate signs related to sodium overload. Recently, Great Britain's National Patient Safety Agency issued recommendations on the use of maintenance fluids and established that any maintenance fluid with a sodium concentration of $0.45 \%$ would put patients at risk. ${ }^{23}$ Maintenance fluids with $0.45-0.9 \% \mathrm{NaCl}$ proved to be safe in studies gathered in a recent systematic review. ${ }^{14}$

Different authors have recommended to measure electrolytes before indicating maintenance fluids and to monitor weight, fluid balance and to strictly check clinical parameters during maintenance fluid infusion. ${ }^{9,12,14}$

The intervention period and the volume of maintenance fluids administered were pragmatic, thus allowing physicians to adjust infused volumes and the time to shift to the oral route. This type of intervention is very similar to the standard of care. Results of specific studies were completely blinded to prevent the Hawthorne effect.

\section{CONCLUSIONS}

This study demonstrated that none of the maintenance fluids under study caused iatrogenic hyponatremia at this rate of infusion over 24 hours. Isotonic maintenance fluids did not reduce serum Na. No differences were observed between isotonic and hypotonic maintenance fluids in terms of the development of hypernatremia, number of days without mechanical ventilation, length of stay in the PICU, and 28 day mortality.

\section{Acknowledgments}

To the members of the PICU and to the Head of the Division of Pharmacy Susana Bengoa at Hospital "Pedro de Elizalde", and to Dr. Fernando Ferrero for his support during the study and the review of the manuscript.

\section{REFERENCES}

1. Holliday MA, Segar ME. The maintenance need for water in parenteral fluid therapy. Pediatrics 1957;19:823-32.

2. Carpenter J, Weinstein S, Myseros J, Vezina G, et al. Inadvertent hyponatremia leading to acute cerebral edema and early evidence of herniation. Neurocrit Care 2007;6(3):195-9.

3. Moritz ML, Ayus JC. Prevention of hospital-acquired hyponatremia: a case for using isotonic saline. Pediatrics 2003;111:227-30.

4. Arieff A, Ayus J, Fraser C. Hyponatremia and death or permanent brain damage in healthy children. BMJ 1992;304:1218-22.

5. Powell KR, Sugarmann LI, Eskanazi AE, Woodin KA, et al. Normalization of plasma arginine vasopressin concentrations when children with meningitis are given maintenance plus replacement fluid therapy. J Pediatr 1990;117(4):515-22.

6. Burrows F, Shutack JG, Crone R. Inappropriate secretion of antidiuretic hormone in a post surgical pediatric population. Intensive Care Med 1983;11:527-31.

7. Thomas TH, Morgan DB. Post-surgical hyponatremia: the role of intravenous fluids and arginine vasopressin. $\mathrm{Br} J$ Surg 1979;66:540-2.

8. Neville K, Verge C, Rosenberg A, O'Meara MW, et al. Isotonic is better than hypotonic saline intravenous rehydration of children with gastroenteritis: a prospective randomized study. Arch Dis Child 2006;91:226-32.

9. Hoorn EJ, Geary D, Robb M, Halperin ML, et al. Acute hyponatremia is related to intravenous fluid administration in hospitalized children: an observational study. Pediatrics 2004;113:1279-84.

10. Eulmesekian PG, Pérez A, Minces P, Bohn D. Hospitalacquired hyponatremia in postoperative pediatrics patients: Prospective observational study. Pediatr Crit Care Med 2010;4(11):479-83.

11. Choong K, Kho ME, Menon K, Bohn D. Hypotonic versus isotonic saline hospitalized children: a systematic review. Arch Dis Child 2006;91:828-35.

12. Jorro Barón F, Balladores C, Carretero P, Lombardo L, et al. Efectos sobre la natremia por la administración de soluciones endovenosas hipotónicas en niños hospitalizados con infección respiratoria aguda baja. Arch Argent Pediatr 2009;107(4):335-9.

13. Álvarez Montañana $P$, Alapont M, Pérez Ocón A, López $\mathrm{PO}$, et al. The use of isotonic fluid as maintenance therapy prevents iatrogenic hyponatremia in pediatrics: A ranomized, controlled open study. Pediatr Crit Care Med 2008;9(6):589-97.

14. Choong K, Arora S, Cheng J, Farrokhyar F, et al. Hypotonic versus isotonic maintenance fluids after surgery forchildren: A randomized controlled trial. Pediatrics 2011;128(5): 857-66.

15. Brazel P, Mc Phee I. Inapropiate secretion of antidiuretic hormone in postoperative scoliosis patients: the role of fluid management. Spine (Phila Pa 1976) 1996;21(6):724-7.

16. Kannan L, Lodha R, Vivekanandhan S, Bagga A, et al. Intravenous fluid regimen and hyponatremia among children: a randomized controlled trial. Pediatr Nephrol 2010;25(11):2303-9.

17. Moritz M, Ayus J. Preventing neurological complications from dysnatremias in children. Pediatr Nephrol 2005;20(12): 1687-700. 
18. Halberthal M, Halperin M, Bohn D. Lesson of the week: Acute hyponatremia in children admitted to hospital. BMJ 2001;322:780-2.

19. Hanna S, Tibby S, Durward A, Murdoch IA. Incidence of hyponatremia and hyponatremic seizures in severe respiratory syncytial virus bronchiolitis. Acta Paediatr 2003;92:430-4.

20. Playfor S. Fatal iatrogenic hyponatremia. Arch Dis Child 2003;88:646-7.
21. Yung M, Keely S. Randomized controlled trial of intravenousmaintenance fluids. J Pediatr Child Health 2009;45(1-2):9-14.

22. Neville K, Sandeman D, Rubinstein A, Henry CM, et al. Prevention of hyponatremia during maintenanceintravenous fluid administration: a prospective randomized study of fluid type versus fluid rate. J Pediatr 2010;156(2):313-9.

23. Suggested template for local development of intravenous fluid guidelines. [Acces: 11/07/2011]. Available at: www. npsa.nhs.uk/health/alerts. 